\title{
NONLINEAR OPTICAL MEASUREMENTS OF ELASTIC CONSTANTS IN NEMATIC LIQUID CRYSTAL
}

\author{
W.K. BAJDECKI* AND M.A. KARPIERZ \\ Institute of Physics, Warsaw University of Technology \\ Koszykowa 75, 00-662 Warszawa, Poland
}

\begin{abstract}
A new method of measurements of elastic constants of nematic liquid crystals by applying the nonlinear optical effect is presented. This method bases on measurements of a value of the reorientation threshold in a liquid crystal layer while the reorientation is induced by the light beam passing through the layer. The obtained results are in a qualitative compatibility with the results obtained by classical methods.
\end{abstract}

PACS numbers: 42:65.-k, 42.70.Df

\section{Introduction}

Liquid crystals have plenty of unique physical, optical, and electrooptical properties and therefore they are important anisotropic materials in modern optoelectronics [1]. New liquid crystalline mixtures and methods of exact measurements of the parameters describing their properties are worked out in laboratories. Among many parameters describing properties of liquid crystals the elastic constants are most important. These constarts are typically determined by observation of the threshold effect of the liquid crystalline reorientation (Frederiks transition effect) due to external electric or magnetic fields [2]. In more advanced methods the measured constants are determined by investigation of the reorientation effect above the threshold (the self-consistent field (SCF) method).

In this paper a new method of measuring the elastic constants in nematic liquid crystals is presented. This method also bases on the reorientation threshold phenomena but the reorientation is caused by the light beam itself. This optical reorientation effect changes the diffraction of the light beam and it is observed in a form of diffraction rings [3]. The value of the threshold light intensity allows us to calculate the elastic constants.

\footnotetext{
*e-mail: bajdecki@if.pw.edu.pl; http://www.if.pw.edu.pl/ opto/
} 


\section{Theory}

The molecular ordering in liquid crystals is caused by a long-distance interaction among liquid crystalline molecules. The initial orientation is induced at the boundaries of the liquid crystalline cell due to the interaction with the surfaces surrounding the liquid crystal and this orientation can be changed by external electric or magnetic field. The strength of the reorientation is described by three fundamental deformations: splay, twist, and bend, and the energy of these deformations are proportional to the elastic constants $K_{11}, K_{22}$, and $K_{33}$, respectively.

Reorientation for a field perpendicular to the initial direction of the liquid crystalline molecules is observed above a certain critical value of this field. This phenomenon is named the Frederiks effect and it also appears for the electric field of the light wave. This situation occurs, e.g. for the nematic liquid crystalline cell in the homeotropic configuration, which is illustrated in Fig. 1. It is assumed

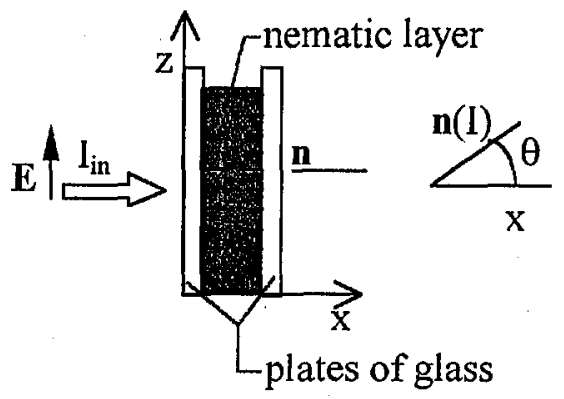

Fig. 1. Light propagation through the nematic film. Director $n$ defines the average direction of the long axis of the liquid crystal molecules.

that the plane optical wave $\boldsymbol{E}=[0,0, E]$ and the absorption and dispersion of the light in the liquid crystalline cell are neglected. Then the equation for the angle $\theta$ describing the direction of orientation of the liquid crystalline molecules is obtained from the Euler-Lagrange equations in the following form [1]:

$$
\begin{aligned}
& \frac{\mathrm{d}^{2} \theta}{\mathrm{d} x^{2}}\left(K_{11} \sin ^{2} \theta+K_{33} \cos ^{2} \theta\right)-\frac{1}{2}\left(\frac{\mathrm{d} \theta}{\mathrm{d} x}\right)^{2}\left(K_{33}-K_{11}\right) \sin 2 \theta \\
& \quad+\frac{\varepsilon_{0} \Delta \varepsilon}{4} E^{2} \sin 2 \theta=0,
\end{aligned}
$$

where $\Delta \varepsilon=n_{\mathrm{e}}^{2}-n_{\circ}^{2}$ is the electric anisotropy, $\varepsilon_{0}$ is the permittivity of the free space, and $K_{11}, K_{33}$ are the elastic constants resulted from the liquid crystalline elastic deformation splay and bend, respectively. The reorientation of molecules leads to the local changes of the medium birefringence axis. The permittivity tensor in the coordinate system rotated at the angle $\theta$ in the $x-z$ plane is equal to

$$
\varepsilon=\left(\begin{array}{ccc}
\varepsilon_{\perp}+\Delta \varepsilon \cos ^{2} \theta & 0 & \Delta \varepsilon \sin \theta \cos \theta \\
0 & \varepsilon_{\perp} & 0 \\
\Delta \varepsilon \sin \theta \cos \theta & 0 & \varepsilon_{\perp}+\Delta \varepsilon \sin ^{2} \theta
\end{array}\right) .
$$


To solve Eq. (1) the boundary conditions on the liquid crystalline boundary surfaces $\theta(0)=\theta(d)=0$ (where $\mathrm{d}$ is the cell thickness) are dictated. If small deformations are assumed $(\sin \theta \approx \theta)$. the relation for the electric field threshold value will be given by

$$
E_{\mathrm{th}}=\frac{\pi}{d}\left(\frac{2 K_{33}}{\varepsilon_{0} \Delta \varepsilon}\right)^{1 / 2} .
$$

Then the corresponding threshold value of the light intensity is equal to

$$
I_{\mathrm{th}}=\langle|\boldsymbol{E} \times \boldsymbol{H}|\rangle=\frac{1}{2}\left|E_{\mathrm{th}}\right|^{2} \sqrt{\frac{\varepsilon \varepsilon_{0}}{\mu \mu_{0}}},
$$

where $\mu_{0}$ and $\mu$ are magnetic permeabilities. For diamagnetic materials $\mu \approx 1$ and at the threshold condition the effective permittivity is equal to $\varepsilon=n_{\circ}^{2}$ (where $n_{\mathrm{o}}$. is the ordinary refractive index) and then the relation for the elastic constant $K_{33}$ is obtained in the following form:

$$
K_{33}=\frac{d^{2} \Delta \varepsilon}{\pi^{2} n_{\mathrm{o}}} \sqrt{\mu_{0} \varepsilon_{0}} I_{\mathrm{th}} .
$$

The accurate solution of Eq. (1) and the exact determination of the field threshold value is possible only with the aid of numerical methods. Figure 2 shows numerical calculations of the angle $\theta$ distribution for different values of the constant external electric field $E$ and for the nematic liquid crystal - pentylocyanobiphenyl (PCB). In calculations the cell thickness $d=48 \mu \mathrm{m}$, the elastic constants $K_{11}=$ $6.8 \times 10^{-12} \mathrm{~N}, K_{33}=10^{-11} \mathrm{~N}$, and the refractive indices $n_{\mathrm{e}}=1.6927$ and $n_{\circ}=$ 1.523 (given for a temperature $t=25.1^{\circ} \mathrm{C}$ ) were taken.

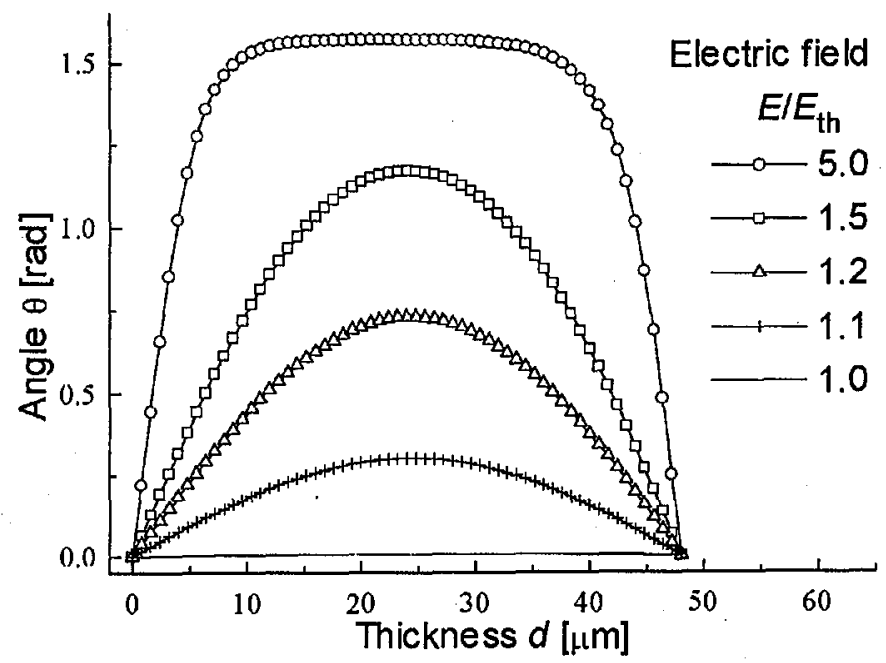

Fig. 2. The distribution of orientation angle $\theta$ for different external electric fields $E$.

For the finite-dimensional light beam the molecules reorientation becomes a function of the distance from the center of the beam. Then the phase structure in the liquid crystal is arisen, which causes the diffraction of the incident light. 
As a result the diffraction pattern with characteristic rings is observed. We assume the Gaussian beam with the electric field intensity distribution in the form $E^{2}(r)=E^{2} \exp \left(-a r^{2}\right)$, where $a=2 / w_{0}^{2}$, and $w_{0}$ is the beam waist. With a small reorientation angle approximation, the distribution of the angle $\theta$ is described by the following relation:

$$
\theta(r, x)=R(r) \sin \left(\frac{\pi x}{d}\right),
$$

which satisfies the strong anchoring conditions $(\theta(r, x)=0$ for $x=0$ and $x=d)$. Then Eq. (1) takes the form [4]

$$
\frac{\mathrm{d}^{2} R}{\mathrm{~d} r^{2}}+\frac{1}{r} \frac{\mathrm{d} R}{\mathrm{~d} r}+\left[b \exp \left(-a r^{2}\right)-\frac{\pi^{2}}{d^{2}}\right] R-\frac{b}{2} \exp \left(-a r^{2}\right) R^{3}=0,
$$

where $b=\left(\varepsilon_{0} \Delta \varepsilon / 2 K\right) E^{2}$ and one-constant approximation $K_{11}=K_{33}=K$ was assumed.

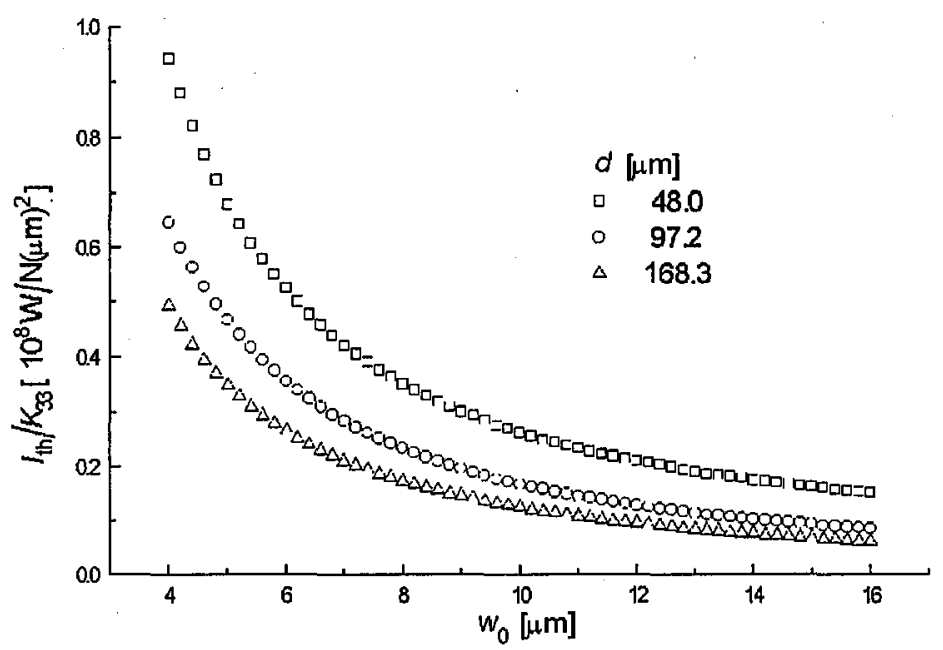

Fig. 3. Threshold light intensity vs. beam waist $w_{0}$ for various thicknesses of the liquid crystalline cell $d$.

The cell thickness relations of the threshold light intensity for various beam waist values obtained from Eq. (7) are illustrated in Fig. 3. In contradistinction to the plane-wave solution the threshold value is a nonlinear function of $1 / d^{2}$ and it has a strong dependence on the beam waist value.

\section{Experimental}

The experimental set-up is presented in Fig. 4. As a light source we used a semiconductor laser SP3509 operating at an IR wavelength $\lambda=842 \mathrm{~nm}$, pig-tailed with the single-mode fiber (SMF). The gradient lens L (SELFOC lens) was used to focus light inside the homeotropic cell of the liquid crystal LC in order to obtain a large power density. The diffraction pattern was observed in the screen plane by a CCD camera. 


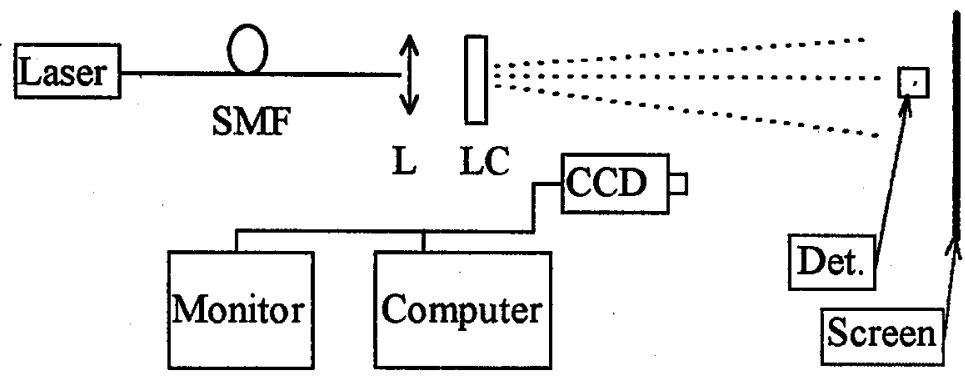

Fig. 4. Experimental set-up for determination of the cell transient characteristics.
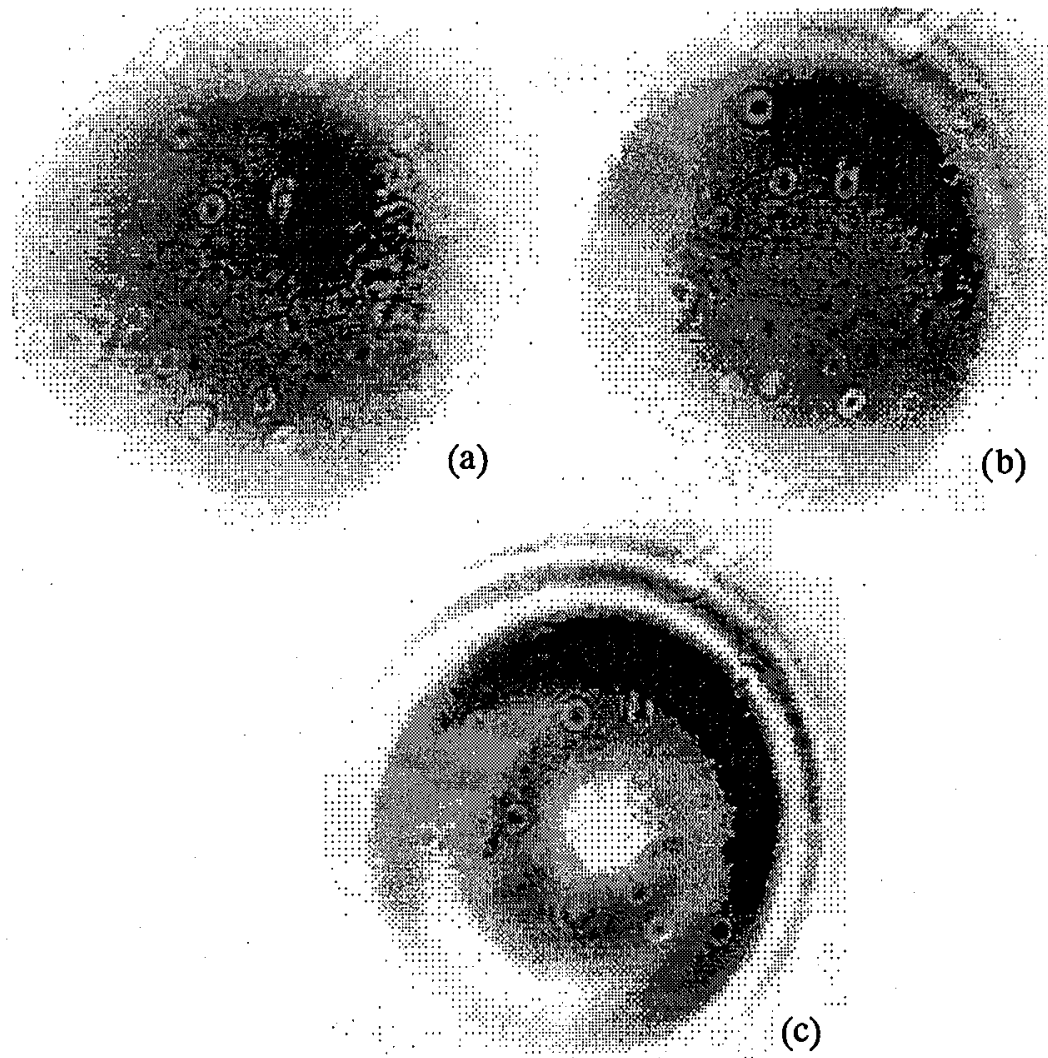

Fig. 5. Diffractional images recorded for PCB cell and $d=97.2 \mu \mathrm{m}$ for various values of the light power emitted by the laser: (a) $15 \mathrm{~mW}$, (b) $21 \mathrm{~mW}$, (c) $24 \mathrm{~mW}$.

Figure 5 shows the diffractional images recorded for the PCB cell with a thickness $d=97.2 \mu \mathrm{m}$. The speckles in these images are caused by an inhomogeneity of the liquid crystalline cell and by impurities of the glass plates limited the cell. The elliptic symmetry of the observed diffractional rings is caused by an angular CCD camera setting related to the optical axis (see Fig. 4). 

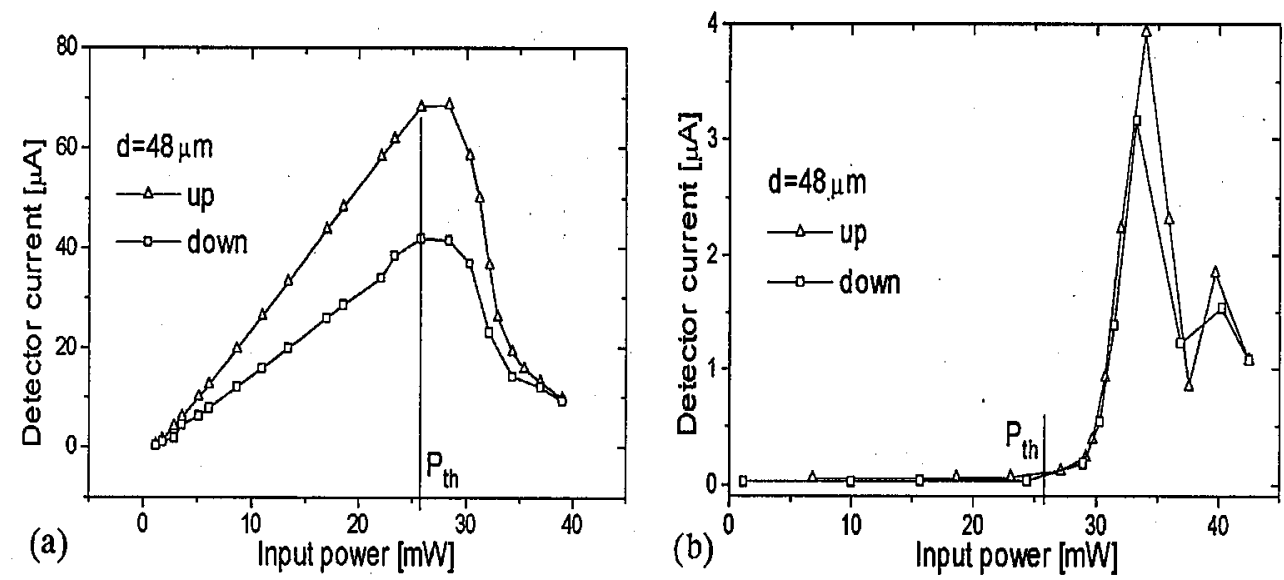

Fig. 6. The LC cell transient characteristics measured by the photodetector placed at the center (a) and in the place of appearance of the first diffraction ring (b).

The relations between the light intensity detected by the photodiode Det. (placed at the center and in the first diffraction ring) and the input light power are illustrated in Fig. 6. These characteristics are used in determination of the power threshold value $\dot{P}_{\text {th }}=\int I_{\text {th }} \mathrm{d} y \mathrm{~d} z$. The measured threshold values were equal to $P_{\text {th }}=26 \mathrm{~mW}$ for a cell thickness $d=48 \mu \mathrm{m}$ and $P_{\text {th }}=17 \mathrm{~mW}$ for a thickness $d=168.3 \mu \mathrm{m}$.

Because the reorientation effect is strongly dependent on the light power density, the accurate determination of the light beam diameter is necessary. The experimental set-up used for measuring the light beam diameter is presented in Fig. 7. The light beam used in previously described experiments is focused inside the diffraction grating $D$ with the index of $4 \mu \mathrm{m}$. The microscopic lens ML transforms the image of the lighted up grating into the CCD camera and the obtained image is shown in Fig. 8. The cross-section of the obtained image was used to fit the envelope of the light beam shape by the Gaussian formula (see Fig. 9). As a result the beam waist $w_{0} \approx(10.3 \pm 0.7) \mu \mathrm{m}$ was estimated. Finally, the value of

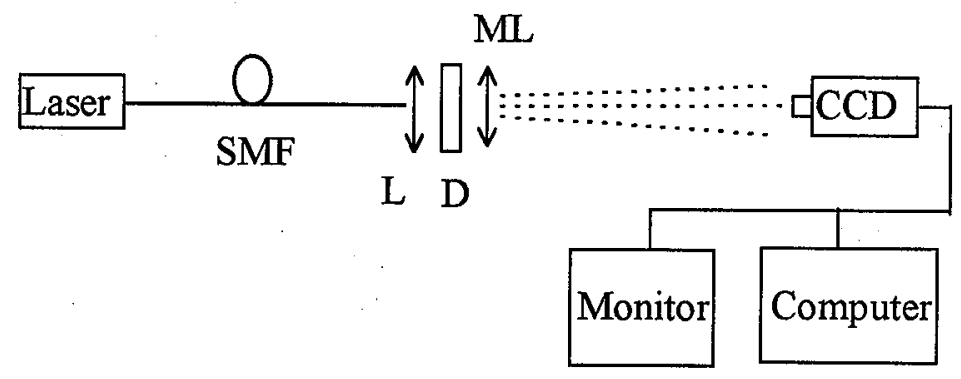

Fig. 7. Experimental set-up for determination of the beam waist. 


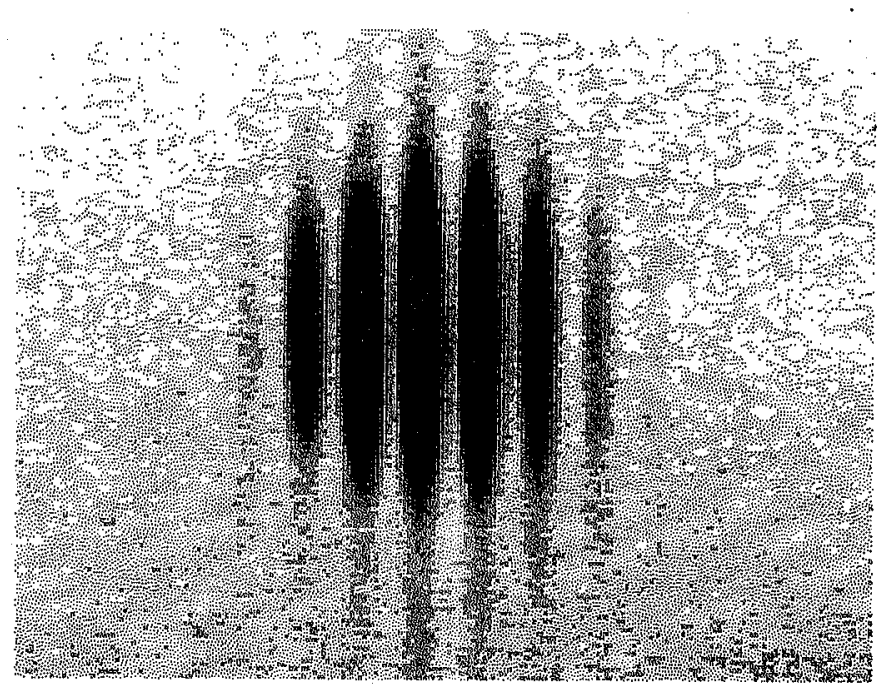

Fig. 8. Image of the lighted up grating.

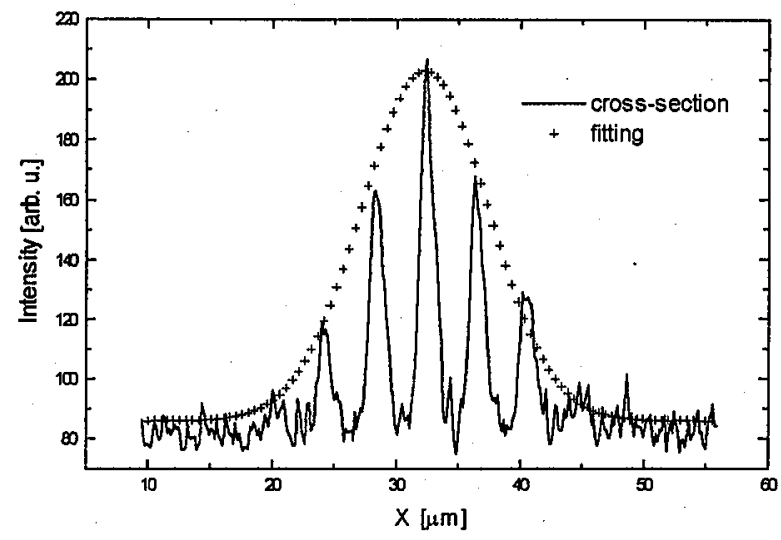

Fig. 9. The image cross-section with fitting of the curve envelope.

the elastic constant $K_{33}=(0.30 \pm 0.08) \times 10^{-11} \mathrm{~N}$ for a cell thickness $d=48 \mu \mathrm{m}$ and the value $K_{33}=(0.42 \pm 0.12) \times 10^{-11} \mathrm{~N}$ for a cell thickness $d=168.3 \mu \mathrm{m}$ were determined. The elastic constant value measured by classical methods (i.e. using constant electric and magnetic fields) was equal to $K_{33}=10^{-11} \mathrm{~N}$.

\section{Conclusions}

In this paper the usability of the nonlinear optical effect in the liquid crystals was analyzed, and the comparison between the experimental results and the values determined in classical methods was shown. The difference between the results 
obtained by classical methods and by the method proposed in this work can be caused by approximations in the theoretical model (among others by neglecting the change of the Gaussian beam diameter inside the LC cell in Eq. (7)) as well as by problematic precision in measurements of threshold power and real beam waist.

Because the qualitative compatibility of the results was achieved, the proposed method of the elastic constants measurement appears as a method giving correct results. The increase in the measuring accuracy can be obtained by the polarization check and by applying a more precise theory of the anchoring molecules on cell walls and the influence of the beam waist on the effect.

This method enables the experimental set-up miniaturization and the measuring process automation. It is a repeatable method and it allows us to obtain many local measurements in the same cell. On the other hand it requires a very precise knowledge of the beam parameters (width, polarization, the focus position inside the cell). Checking the polarization at the cells configured planarly we can measure the force constant $K_{22}$ connected with the twist deformation.

\section{Acknowledgments}

The authors would like to express their gratitude to Prof. Jerzy Kędzierski for his technical support and results of classical measurements of the liquid crystalline elastic constants. This work was partially supported by the Committee for Scientific Research under the grant No. 8 T11D 01915.

\section{References}

[1] I.C. Khoo, S.T. Wu, Optics and Nonlinear Optics of Liquid Crystals, World Scientific Publ., Singapore 1993.

[2] J. Kędzierski, Z. Raszewski, J. Rutkowska, T. Opara, J. Zieliński, J. Żmija, R. Dąbrowski, Mol. Cryst. Liq. Cryst. 249, 119 (1994).

[3] M.A. Karpierz, A.W. Domański, M. Sierakowski, M. Świłło, T.R. Woliński, in: Proc. IEEE Instrum. Meas. Technol. Conf. IMTC '97, Ottawa (Canada) 1997, IEEE, 1997, p. 711.

[4] W.K. Bajdecki, M.A. Karpierz, in: Optical Fibers and their Applications VI, Eds. J. Dorosz, R.S. Romianiuk, in series Proc. SPIE, Vol. 3731, in press. 